\title{
Myocyte hypertrophy: the long and winding RhoA'd
}

Commentary

See related article

this issue, pages 1627-1634

\section{Toren Finkel}

Address correspondence to: National Institutes of Health, Cardiology Branch, Building 10/7B-15, 10 Center Drive, Bethesda, Maryland 20892-1650, USA. Phone: (301) 402-4081; Fax: (301) 402-0888; E-mail: finkelt@gwgate.nhlbi.nih.gov.

It seems we live in increasing complexity, and the world of signal transduction is no different. The orderly, linear pathways that just a few years ago appeared to link the cell surface to the nucleus have led to a twisting road with an array of intersecting and overlapping cascades. The fascinating study by Sah and colleagues in this issue (1) adds wonderfully to the present understanding of our location on this changing roadmap.

Cardiomyocytes have a limited repertoire of responses. Faced with a number of seeming diverse insults ranging from ischemia/reperfusion, adrenergic stimulation, or hemodynamic overload, myocytes respond by undergoing cellular hypertrophy. This process appears to be repeated faithfully when neonatal myocytes in culture are exposed to a variety of ligands or mechanical forces. Several years ago, in a pioneering study from the laboratory of Ken Chien, the path by which cardiomyocytes undergo hypertrophy began to unravel. These investigators expressed an activated form of the Ras oncogene in neonatal myocytes and subsequently noted that the cells underwent a classical hypertrophic response, including the reexpression of embryonic genes such as atrial natriuretic factor (ANF), an increase in cell size, and the production of sarcomeric structures (2).

Although these studies demonstrated that Ras was capable of stimulating hypertrophy, they left unanswered which proteins were downstream of Ras. Many of Ras effects in proliferating cells result from its ability to form a

\section{Figure 1}

The potential role of small GTPases in cardiac hypertrophy. Small GTPases are shown in blue, their immediate and more distal effectors are shown in green and the potentially relevant targets in yellow. Although drawn as linear pathways, each cascade is capable of multiple intersections. As an example, the small GTPases can interact with each other and MEKK1 can potentially participate in more than one pathway. molecular complex with the protein kinase c-Raf and subsequently to activate the family of mitogen-activated protein kinases (MAPK). However, it soon became clear that in cardiomyocytes, activation of MAPK could not be the sole pathway for hypertrophy. Several reports noted that activation of MAPK did not always correlate with induction of hypertrophy (3) and that expression of dominant negative forms of either c-Raf or MAPK could not completely abolish ligand-stimulated hypertrophy $(4,5)$. In addition, there was no evidence of increased MAPK activity in transgenic mice that overexpressed an activated Ras gene (6), although there was significant ventricular hypertrophy.

In the search for other Ras effectors, a considerable amount of attention has centered lately on the Rho family of small GTPases, a family that

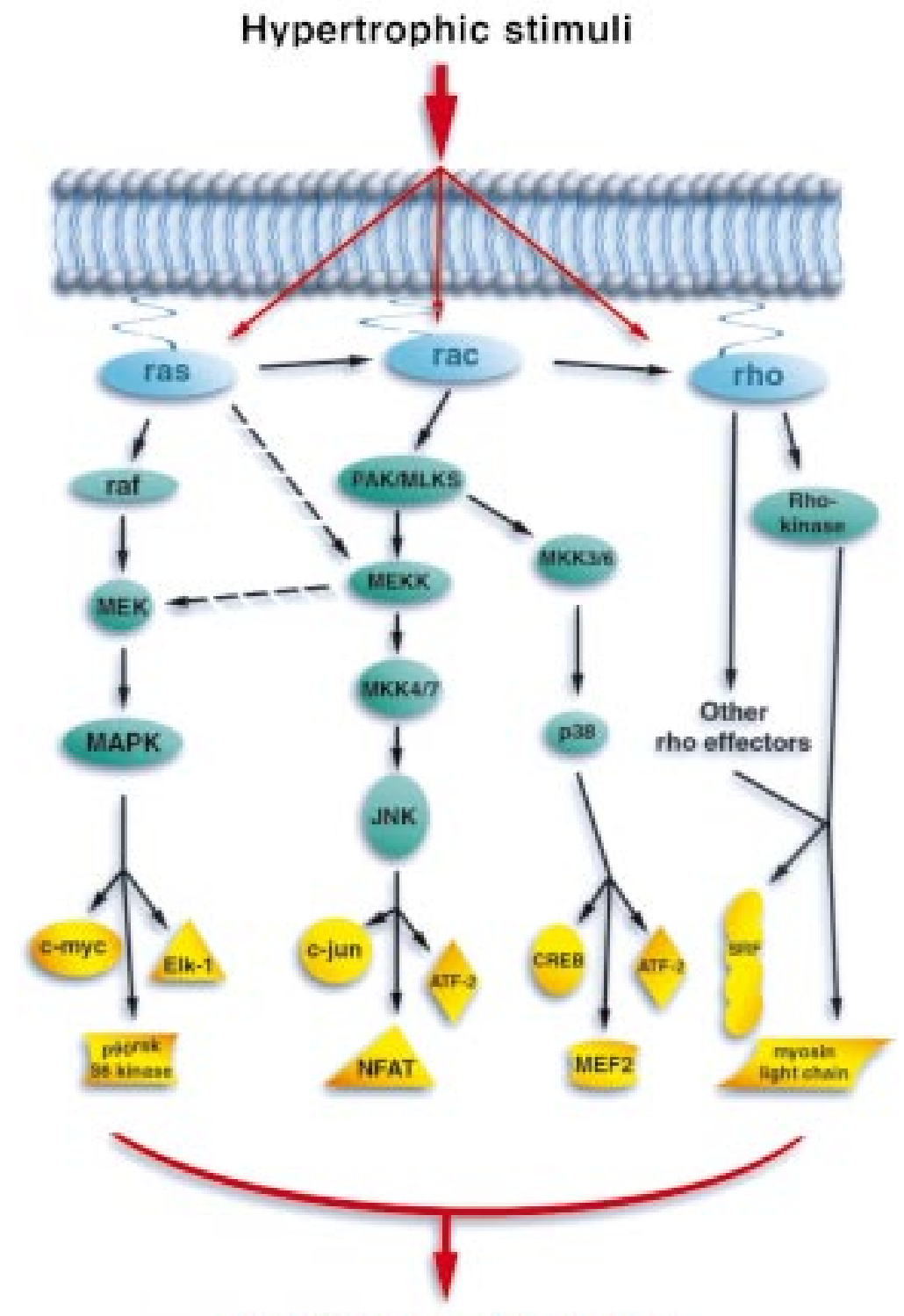

Hypertrophic response 
includes Rac1, Cdc42, and RhoA. The interest in this family exploded after the demonstration that these proteins regulate cytoskeleton reorganization and that they synergize with Ras or Raf in growth and transformation assays (7). A role for the Rho GTPases in myocyte hypertrophy is supported by several recent studies demonstrating the effects of activated and dominant negative forms of RhoA on hypertrophic target gene expression (8-11). The effects of RhoA on the morphological aspects of hypertrophy were less clear, with recent reports giving conflicting results $(9,10)$. Similarly, expression of an activated form of Rac1 appeared to stimulate the hypertrophic program while expression of a dominant negative Rac gene product was inhibitory $(11,12)$.

In fibroblasts there is a hierarchy of small GTP-binding proteins, with Ras able to stimulate $\mathrm{Cdc} 42$, which can then stimulate Rac, which can in turn stimulate RhoA (7). Whether a similar hierarchy exists in other cells is unknown. Both Cdc42 and Rac can regulate the activity of c-Jun $\mathrm{N}$-terminal kinases (JNK) and the p38 kinase family. Ras proteins can also weakly stimulate JNK, perhaps by activating Rac or alternatively by directly activating MEKK1 (see Figure 1). A flurry of recent reports have implicated the activation of JNK and p38 in the hypertrophic response $(6,13-17)$. However, simultaneous activation of JNK and p38 appears not to lead to hypertrophy but instead to cell death $(15,18)$. These results may provide insight into the molecular pathway that could explain the point at which an adaptive hypertrophic response becomes a maladaptive myopathy.

Since RhoA does not usually stimulate JNK or p38, how does it contribute to hypertrophy? One explanation is by regulating the activity of transcription factors such as the serum response factor. Alternatively, in smooth muscle cells, RhoA, through Rho-kinase (one of its downstream effectors), appears to regulate the phosphorylation status of myosin light chain (7). The role of myosin light chain phosphorylation in cardiac muscle cells is unclear. Interestingly, however, mutation of the cardiac myosin regulatory light chain, near the site of phosphorylation, produces significant cardiac hypertrophy in patients who inherit the mutant allele (19).

Given this background, it is somewhat surprising that there was no evidence of ventricular hypertrophy when Sah and colleagues overexpressed RhoA in the myocardium (1). Instead the authors observed atrial enlargement and a greatly depressed heart rate. Although these results might question the validity of the widely used in vitro neonatal cell model, they more likely reflect the embryonic pattern of expression of RhoA when it is coupled with the $\alpha$-myosin heavy chain promoter. The observed pattern of ventricular dilation may merely be secondary to the atrial phenotype or, alternatively, it is possible that high RhoA activity in the ventricle may produce an apoptotic response. Interestingly, the observed bradycardia may relate to another newly described downstream effector of RhoA - the delayed rectifier channel (20). It appears likely that in the near future other small GTPases will be implicated in cardiac channel regulation.

The small GTPases, nature's most versatile switches, appear to play an everexpanding and interconnecting role in myocyte biology. Their ultimate function in hypertrophy and excitability requires further experimentation; going down that long and winding road, Sah and colleagues (1) remind us that there will undoubtedly be some wonderful twists and turns.

\section{Acknowledgment}

This commentary was written by the author in his private capacity. The views expressed in this article do not necessarily represent the views of NIH,DHHS, nor the United States.

1. Sah, V.P., et al. 1999. Cardiac-specific overexpression of RhoA results in sinus and atrioventricular nodal dysfunction and contractile failure. J. Clin. Invest. 103:1627-1634.

2. Thorburn, A., et al. 1993. HRas-dependent pathways can activate morphological and genetic markers of cardiac muscle cell hypertrophy. J. Biol. Chem. 268:2244-2249.

3. Post, G.R., Goldstein, D., Thuerauf, D.J., Glembotski, C.C., and Brown, J.H. 1996. Dissociation of p44 and p42 mitogen-activated protein kinase activation from receptor-induced hypertrophy in neonatal rat ventricular myocytes. J. Biol. Chem. 271:8452-8457.

4. Thorburn, J., McMahon, M., and Thorburn, A. 1994. Raf-1 kinase activity is necessary and suffi- cient for gene expression changes but not sufficient for cellular morphology changes associated with cardiac myocyte hypertrophy. J. Biol. Chem. 269:30580-30586

5. Thorburn, J., Frost, J.A., and Thorburn, A. 1994. Mitogen-activated protein kinases mediate changes in gene expression, but not cytoskeletal organization associated with cardiac muscle cell hypertrophy. J. Cell Biol. 126:1565-1572.

6. Ramirez, M.T., Sah, V.P., Zhao, X.L., Hunter, J.J., Chien, K.R., and Brown, J.H. The MEKK-JNK pathway is stimulated by $\alpha_{1}$-adrenergic receptor and Ras activation and is associated with in vitro and in vivo cardiac hypertrophy. 1997. J. Biol. Chem. 272:14057-14061.

7. VanAelst, L., and D'Souza-Schorey, C. 1997. Rho GTPases and signaling networks. Genes Dev 11:2295-2322.

8. Sah, V.P., Hoshijima, M., Chien, K.R., and Brown, J.H. 1996. Rho is required for $G \alpha_{q}$ and $\alpha_{1}$-adrenergic receptor signaling in cardiomyocytes. J. Biol. Chem. 271:31185-31190.

9. Hoshijima, M., Sah, V.P., Wang, Y., Chien, K.R., and Brown, J.H. 1998. The low molecular weight GTPase Rho regulates myofibril formation and organization in neonatal rat ventricular myocytes. J. Biol. Chem. 273:7725-7730.

10. Thorburn, J., Xu, S., and Thorburn, A. 1997. MAP kinase- and Rho-dependent signals interact to regulate gene expression but not actin morphology in cardiac muscle cells. EMBO J. 16:1888-1900.

11. Aikawa, R., et al. 1999. Rho family small G proteins play critical roles in mechanical stressinduced hypertrophic responses in cardiac myocytes. Circ. Res. 84:458-466.

12. Pracyk, J.B., et al. A requirement for the rac1 GTPase in the signal transduction pathway leading to cardiac myocyte hypertrophy. 1998. J. Clin. Invest. 102:929-937.

13. Zechner, D., Thuerauf, D.J., Hanford, D.S., McDonough, P.M., and Glembotski, C.C. 1997. A role for the p38 mitogen-activated protein kinase pathway in myocardial cell growth, sarcomeric organization, and cardiac-specific gene expression. J. Cell Biol. 139:115-127.

14. Wang, Y.B., et al. 1998. Cardiac muscle cell hypertrophy and apoptosis induced by distinct members of the p38 mitogen-activated protein kinase family. J. Biol Chem. 273:2161-2168.

15. Wang, Y.B., et al. 1998. Cardiac hypertrophy induced by mitogen-activated protein kinase kinase 7, a specific activator for c-jun NH2-terminal kinase in ventricular muscle cells. J. Biol. Chem. 273:5423-5426.

16. Clerk, A., Michael, A., and Sugden, P.H. 1998. Stimulation of the p38 mitogen-activated protein kinase pathway in neonatal rat ventricular myocytes by the $G$ protein coupled receptor agonists, endothelin-1 and phenylephrine: A role in cardiac myocyte hypertrophy? J. Cell Biol. 142:523-535.

17. Nemoto, S., Sheng, Z., and Lin, A. 1998. Opposing effects of jun kinase and p38 mitogen-activated protein kinases on cardiomyocyte hypertrophy. Mol. Cell. Biol. 18:3518-3526.

18. Adams, J.W., et al. Enhanced G $\alpha q$ signaling: A common pathway mediates cardiac hypertrophy and apoptotic heart failure. 1998. Proc. Nat. Acad. Sci. USA. 95:10140-10145.

19. Poetter, K., et al. 1996 Mutations in either the essential or regulatory light chains of myosin are associated with a rare myopathy in human heart and skeletal muscle. Nat. Genet. 13:63-69.

20. Cachero, T.G., Morielli, A.D., and Peralta, E.G. The small GTP-binding protein RhoA regulates a delayed rectifier potassium channel. 1998. Cell. 93:1077-1085. 